\title{
I farmaci biologici in Nefrologia
}

\author{
Roberta Fenoglio, Dario Roccatello
}

CMID, Centro di Ricerche di Immunopatologia e Documentazione su Malattie Rare (Centro di Coordinamento della Rete delle Malattie Rare del Piemonte e della Valle d’Aosta) e SCDU Nefrologia e Dialisi, Ospedale S. Giovanni Bosco e Università degli Studi di Torino, Torino

\begin{abstract}
Biologic therapeutic agents in nephrology
Biological drugs represent one of the most important therapeutic novelties of the last twenty years in many areas including Nephrology. Their use was initially limited to the transplantation field, but now it is extended to the treatment of many glomerular diseases. The selectivity of these drugs should ensure a decrease in the side effects associated with conventional immunosuppressive therapy and an improvement in patient compliance. These aspects, together with the identification of previously unknown pathogenic mechanisms of many glomerular nephropathies, resulted in an increased use of biologic drugs even in kidney diseases. Rituximab was introduced in the mid ' 90 s and it was the pioneering drug among biologic agents in Nephrology. Since then, clinincians have become more and more interested in this class of drugs, and the potential of these agents is on the rise. Currently unresolved issues include both safety and cost/benefit ratio of their long-term use. Undoubtedly, it is the most significant progress that has been made from a therapeutic point of view in recent years.
\end{abstract}

Keywords: Biologic drugs, Anti-CD20 monoclonal antibody, Rituximab, Therapy of glomerulonephritis

\section{Introduzione}

I farmaci biotecnologici rappresentano una delle novità terapeutiche più rilevanti degli ultimi venti anni e hanno aperto frontiere di intervento farmacologico innovativo. Questi trattamenti hanno rivoluzionato la terapia di una serie di condizioni patologiche in numerose discipline, in particolare l'oncologia e la reumatologia. A partire dagli anni '90, i farmaci biologici si sono affacciati anche al mondo nefrologico. Le indicazioni, inizialmente ristrette all'ambito trapiantologico, si sono successivamente estese al trattamento di molte patologie glomerulari (1). A livello globale, l'uso dei farmaci biologici è quasi raddoppiato negli ultimi dieci anni, passando dai 6 miliardi di Defined Daily Dose (DDD) del 2003 agli 11.9 miliardi del 2013.

Si definiscono biotecnologici quei farmaci, ottenuti mediante la tecnica del DNA ricombinante, che sono bersaglioselettivi (singola struttura: recettore, proteina, sequenza di

Accepted: October 31, 2015

Published online: November 23, 2015

Indirizzo per la corrispondenza:

Prof. Dario Roccatello

Ospedale S. Giovanni Bosco

Piazza del Donatore di Sangue 3

10154 Torino

dario.roccatello@unito.it
DNA) e, dunque, associati a limitati effetti collaterali.

La tecnica per ottenere gli ibridomi fu ideata nel 1975 da Georges Köhler e Cesar Milstein. Tale scoperta consenti loro di ricevere, nel 1984, il premio Nobel per la Medicina senza che fosse ancora chiaro il potenziale di questa tecnica ai fini della produzione di anticorpi con attività terapeutica. L'ibridoma convenzionale si ottiene tramite fusione di un linfocita $B$, deputato alla produzione di anticorpi, con una cellula di una linea tumorale immortalizzata derivata da un tumore dei linfociti B. Dalla popolazione eterogenea di cellule che derivano da questa fusione vengono selezionati gli elementi che presentano le caratteristiche di entrambe le cellule di origine, in grado di produrre un particolare anticorpo e di proliferare indefinitamente. Gli ibridomi mantengono, quindi, la capacità dei linfociti di produrre anticorpi specifici, ma assumono la proprietà, tipica delle cellule mielomatose, di dividersi indefinitamente, garantendo un'elevata produzione di anticorpo. Le prime applicazioni terapeutiche degli anticorpi monoclonali sono state fatte con anticorpi di derivazione murina. L'efficacia di questi anticorpi era inizialmente limitata da fattori fisiologici, tra cui l'incostante riconoscimento dell'epitopo umano (per esempio, un recettore cellulare) da parte dell'anticorpo murino e la loro ridotta emivita dovuta all'insorgere di una risposta anticorpale dell'ospite verso agenti farmacologici riconosciuti come estranei dal sistema immunitario. Questo ostacolo all'utilizzo degli anticorpi monoclonali in terapia è stato superato con I'utilizzo della tecnologia del DNA ricombinante che ha consentito, attraverso tappe successive, la ri- 


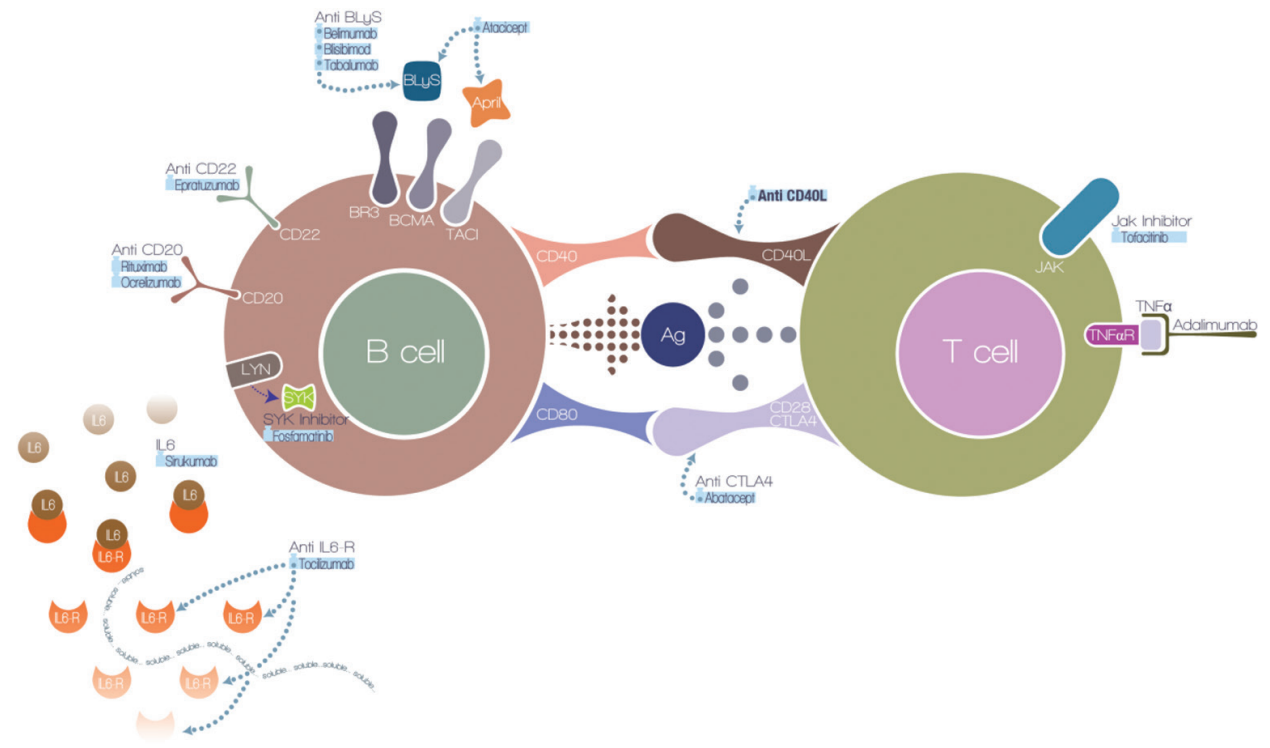

Fig. 1 - Principali target dei farmaci biologici utilizzati in Nefrologia.

duzione dell'immunogenicità degli Ab murini dall' $80 \%$ al 5\%.

In prima battuta sono stati generati anticorpi costituiti da una regione variabile murina (33\%) e da una regione costante umana, cosiddetti chimerici. Queste modificazioni si sono ulteriormente evolute fino a ottenere anticorpi in cui la proteina murina è rappresentata solo in quelle parti dell'anticorpo che interagiscono con l'antigene (regione ipervariabile, $5-10 \%)$ mentre il resto della immunoglobulina è umano. $\mathrm{Si}$ parla di anticorpi umanizzati. Infine, grazie alle tecnologie rese disponibili dalla creazione di animali transgenici, l'informazione genetica per la sintesi di immunoglobuline umane è stata trasferita nel topo. Si è creato un animale capace di produrre, dopo l'esposizione all'antigene, anticorpi identici a quelli umani.

Una differente manipolazione ha consentito di coniugare la regione costante di un'IgG1 con una specifica componente molecolare, per esempio un recettore cellulare, generando una proteina di fusione.

Il rapido incremento del numero di anticorpi monoclonali e le variazioni delle caratteristiche di composizione (murino/ umano) hanno reso necessaria la formulazione di una specifica nomenclatura per l'identificazione della molecola. II suffisso mab, utilizzato per identificare l'anticorpo monoclonale, è preceduto dall'identificazione della specie animale predominante (xi, chimera, zu, umanizzato, $\mathbf{u}$, umano) e dal tipo di patologia target (li o lim, impiego in patologia immune, tu, impiego in patologia tumorale).

La selettività di questi farmaci ha consentito una notevole riduzione degli effetti collaterali comunemente associati alle terapie immunosoppressive convenzionali e un miglioramento della compliance dei pazienti. Questi aspetti, associati all'identificazione di nuovi meccanismi immunopatogenetici delle nefropatie glomerulari, hanno consentito un utilizzo crescente dei farmaci biotecnologici in ambito nefrologico (Fig. 1).

\section{Deplezione delle cellule B}

Il Rituximab (RTX) è sicuramente l'agente biologico più utilizzato nell'ambito del trattamento delle nefropatie immunomediate (2).

Una delle indicazioni nefrologiche più consolidate per l'utilizzo dell'anticorpo monoclonale anti-CD20 è rappresentata dalle vasculiti ANCA-correlate.

Nel 2011, la FDA ha approvato l'impiego del RTX nel trattamento delle micropoliangioiti sistemiche. Molti studi aperti ne hanno documentato effetti positivi sia impiegato da solo che in associazione ad altri immunosoppressori $(3,4)$.

Questi profili favorevoli sono stati confermati dai due studi randomizzati, il RITUXIVAS (5) e il RAVE (6), che hanno stabilito definitivamente i benefici clinici del farmaco nelle micropoliangioiti.

II RITUXIVAS ha confrontato l'utilizzo del RTX in associazione a boli di metilprednisolone e ciclofosfamide in due somministrazioni endovenose o per os per 3-6 mesi seguita da azatioprina come terapia di mantenimento. La percentuale di remissione è risultata sovrapponibile nei due gruppi a parità di effetti collaterali.

II RAVE è uno studio multicentrico in cui i pazienti venivano trattati con steroide ( 6 mesi) e RTX ( 375 mg/mq, 4 somministrazioni) o steroide e ciclofosfamide, seguita, per il mantenimento, da azatioprina. II RTX ha ottenuto la remissione nel $64 \%$ dei casi (versus 53\% con la ciclofosfamide) ed è risultato superiore nell'indurre la remissione nei pazienti con malattia recidivante. Gli effetti collaterali registrati sono stati sovrapponibili.

Studi successivi hanno confermato anche nel lungo periodo gli effetti favorevoli del RTX nel trattamento delle vasculiti.

Rimangono controverse l'indicazione e le modalità di somministrazione del RTX come terapia di mantenimento. Un'analisi retrospettiva in cui la somministrazione del farmaco veniva proseguita dopo la remissione (con frequenza circa 
quadrimestrale) ha mostrato risultati favorevoli, confermati da studi successivi (7).

È in corso lo studio RITAZAREM che confronta l'efficacia di RTX (1000 mg ogni 4 mesi) versus azatioprina (o metotrexate nei pazienti intolleranti) nel mantenere la remissione.

La dose adeguata di mantenimento rimane da definire.

Importante è il ruolo del RTX anche nel trattamento della nefrite lupica. II primo report di terapia con RTX nel LES risale al 2002. Da allora, è stato ampiamente utilizzato in off-label. II RTX agisce attraverso tre meccanismi principali, che includono la citotossicità cellulo-mediata, la citotossicità complemento-dipendente e l'apoptosi, che garantiscono, nell' $80 \%$ dei pazienti, una deplezione delle cellule B per 6-9 mesi. II ripopolamento delle cellule $B$ coinvolge principalmente un subset di linfociti $\mathrm{B}$ naive con modalità simili al ripopolamento che si osserva dopo il trapianto di midollo. La ricostituzione di una popolazione naive supporta il ruolo di questo agente biologico nel trattamento del LES (8). Su questa base sono stati condotti studi che, impiegando l'anti-CD20 associato a una piccola dose di potenziamento di ciclofosfamide, hanno evidenziato buoni effetti sulle manifestazioni sistemiche (9).

Un trial di fase I/II (EXPLORER) (10) nel lupus extrarenale non ha confermato la superiorità del farmaco rispetto al placebo quando aggiunto a un trattamento convenzionale. Anche il successivo studio LUNAR (fase III) (11), in cui pazienti con nefrite lupica di classe III e IV venivano trattati con RTX o placebo in aggiunta alla terapia convenzionale, non ha raggiunto l'end-point primario (remissione completa o parziale a un anno).

Trattamento di base, terapie concomitanti e fattori etici sono stati considerati elementi critici nel determinare outcome diversi negli studi controllati e non. Gli studi aperti erano rivolti a pazienti non-responder o intolleranti ai farmaci immunosoppressori convenzionali non arruolabili negli studi randomizzati controllati.

Nonostante l'apparente fallimento dei trial controllati, molti clinici hanno continuato a utilizzare il farmaco sulla base di esperienze aneddotiche. Studi recenti hanno, peraltro, confermato l'efficacia del trattamento del RTX sia nel contenimento delle manifestazioni sistemiche che nel risparmio/sospensione degli altri farmaci immunomodulanti associati (12-15).

L'impiego del RTX ha consentito dei buoni risultati anche nel trattamento di molte delle manifestazioni sistemiche della crioglobulinemia mista (CM); in particolare, il farmaco si è dimostrato efficace in circa il $90 \%$ delle glomerulonefriti $(16,17)$.

In uno studio multicentrico pubblicato nel 2012 (16), pazienti non eleggibili o refrattari alla terapia antivirale venivano trattati con RTX o con terapia immunosoppressiva convenzionale. I risultati hanno evidenziato una superiorità del RTX rispetto al trattamento immunosoppressivo convenzionale. Come in altre patologie, la durata della risposta non è ancora definitivamente chiarita, tuttavia recidive a breve termine sono riportate in una minoranza di pazienti. Nel tentativo di ritardare eventuali flare, è stata proposta l'aggiunta di ulteriori due dosi del farmaco 1 e 2 mesi dopo un ciclo standard di 4 somministrazioni settimanali (17). II ritrattamento con RTX delle recidive si è dimostrato efficace nella maggior parte dei casi (18).

La nefropatia membranosa idiopatica è caratterizzata dalla deposizione di immunocomplessi lungo la membrana basale glomerulare che, nella maggior parte dei casi, sono l'effetto della formazione di autoanticorpi diretti contro il recettore A2 della fosfolipasi di tipo M. II livello di questi autoanticorpi è un indicatore sensibile e specifico di malattia. La scoperta di questi autoanticorpi ha potenziato il razionale dell'utilizzo del RTX nel trattamento di questa patologia (19). Numerosi studi non controllati hanno riportato una remissione completa o parziale dopo terapia rispettivamente nel $15-20 \%$ e nel $35-40 \%$ dei pazienti. La variabilità di questi risultati può essere interpretata come secondaria ai diversi schemi terapeutici utilizzati e alle diverse caratteristiche iniziali dei pazienti. II RTX è stato utilizzato anche come terapia di mantenimento nei pazienti con remissione della sindrome nefrosica successiva al trattamento con inibitori delle calcineurine ma "dipendenti" dal farmaco. L'efficacia del RTX può essere limitata dall'entità della proteinuria, che può determinare una perdita urinaria del farmaco, e dallo sviluppo di anticorpi anti-RTX (20). Alcuni studi recenti propongono l'utilizzo in sequenza di tacrolimus-RTX con buoni risultati sia in termini di remissione che di recidive. È in corso un trial che confronta l'efficacia e la sicurezza di questa terapia con il protocollo di Ponticelli modificato (steroide e ciclofosfamide) (21). Al momento, le dosi ottimali, la frequenza delle somministrazioni e gli effetti a lungo termine rimangono da definire.

In letteratura, stanno aumentando le segnalazioni a favore del trattamento con RTX di pazienti con glomerulonefrite a lesioni minime steroido-resistenti $(22,23)$. Più discusso, invece, è il ruolo del farmaco nel trattamento delle GSFS, dove i risultati sembrano essere meno favorevoli, particolarmente nell'adulto (24).

L'efficacia del RTX è stata riportata anche in casi sporadici di glomerulonefrite fibrillare (25) e di glomerulonefrite da anticorpi anti-membrana basale (26). Infine, è, attualmente, in corso un trial (NCT00498368) finalizzato a valutare l'efficacia del RTX nel trattamento della nefropatia IgA.

Negli ultimi anni, sono stati proposti altri anticorpi monoclonali anti-CD20, caratterizzati da un'affinità superiore, da una ridotta immunogenicità e da un'incrementata citotossicità. L'Ocrelizumab (anti-CD20 umanizzato) è stato testato nella nefropatia lupica, ma lo studio è stato interrotto per un elevato numero di complicanze (27). Nel trattamento del LES con deplezione delle cellule $B$ è stato anche proposto l'utilizzo dell'Epratuzumab $(28,29)$. Si tratta di un anticorpo monoclonale anti-CD22, una proteina transmembrana espressa sui linfociti B maturi capace di influenzare la loro migrazione e la loro attivazione. L'Epratuzumab, in particolare, sarebbe in grado di ridurre il CD22 minimizzando la distruzione delle cellule B (45\% versus il $90 \%$ del RTX) e l'impatto sul sistema immunitario. I 2 studi randomizzati ALLEVIATE 1 e ALLEVIATE 2 (30) hanno 
documentato qualche efficacia del farmaco, somministrato in associazione alla terapia standard in pazienti con forme severe di LES, in termini di riduzione del BILAG-SCORE e di riduzione del dosaggio dello steroide. Questi risultati incoraggianti hanno consentito lo sviluppo di uno studio di fase III (EMBODY-STUDY) finalizzato a valutare l'efficacia a lungo termine dell'Epratuzumab nel LES (31).

\section{Blocco dell'attivazione delle cellule B}

II Belimumab è un anticorpo monoclonale umanizzato (Ig)$\mathrm{G} 1 \lambda$, che agisce bloccando il legame della forma solubile del Blys, conosciuto anche come fattore attivante i linfociti B (BAFF), ai recettori $\mathrm{TACl}, \mathrm{BCMS}$ e BAFF3, presenti sulla superficie degli stessi linfociti. In presenza di un eccesso di BAFF (IL-1, TNF- $\alpha$, IFN), i linfociti B autoreattivi possono sopravvivere favorendo la comparsa di plasmacellule secernenti auto-anticorpi implicati nella patogenesi delle malattie autoimmunitarie. I pazienti con LES hanno elevati livelli di Blys nel siero e nel liquor e gli elevati livelli sierici sono associati agli anticorpi anti-DNA e all'attività della malattia. Partendo da questo presupposto, è stato ipotizzato che l'inibizione del BAFF/Blys potesse avere delle implicazioni terapeutiche nel LES. In particolare, il farmaco è stato valutato attraverso alcuni studi di fase I-II, a cui sono seguiti i due studi di fase 3 randomizzati in doppio cieco controllati con placebo (BLISS-76, BLISS-52) $(32,33)$. Questi studi non includevano pazienti con coinvolgimento renale severo, ma un'analisi post-hoc ha suggerito qualche effetto favorevole del Belimumab nei pazienti con nefrite lupica attiva. Belimumab è stato, quindi, il primo farmaco registrato per il trattamento del LES dopo oltre 50 anni; il suo impiego è, attualmente, indicato come terapia aggiuntiva nei pazienti adulti con LES attivo, con autoanticorpi e con un alto grado di attività della malattia nonostante la terapia standard.

Sono attualmente in corso due trial per valutare l'impiego del Belimumab nel trattamento della glomerulonefrite membranosa idiopatica e delle vasculiti (34). II secondo è uno studio di fase III per la valutazione dell'efficacia e della sicurezza del farmaco somministrato in associazione all'azatioprina nel mantenimento (BREVAS study). Altri due agenti anti-BAFF hanno suscitato I'interesse nefrologico. Si tratta del Tabalumab e del Blisimod. Isenberg et al (35) hanno recentemente pubblicato uno studio di fase III in cui sono state valutate l'efficacia e la sicurezza del Tabalumab somministrato per via sottocutanea in pazienti con LES i cui risultati non hanno confermato le aspettative. È ancora in corso, invece, lo studio BRILLIANT-SC, finalizzato a valutare l'efficacia e la sicurezza del Blisibimod nel trattamento della glomerulonefrite IgA.

Infine, l'Atacicept, una proteina di fusione in grado di inibire BAFF e APRIL, è stato valutato in un trial di pazienti con nefrite lupica. Lo studio è stato interrotto per la comparsa di un'ipogammaglobulinemia severa e di complicanze infettive (36).

Recentemente, è stata proposta un'ulteriore strategia di interferenza sull'attivazione dei linfociti B attraverso l'inibizione di spleen tyrosine kinase (Syk) e protein tyrosine kinase (JAK) (37). Le tyrosine kinase sono coinvolte nella trasduzione di numerosi segnali e sembrano avere un ruolo regolatore in alcune risposte allergiche, infiammatorie e autoimmunitarie. II fosfamatinib e il tofacitinib (38), inibitori selettivi rispettivamente di Syk e JAK, trovano, quindi, delle potenziali applicazioni nel trattamento dell'artrite reumatoide e nel LES. Alcuni studi sperimentali hanno dimostrato che gli inibitori di Syk sono in grado di prevenire lo sviluppo della nefrite nei pazienti affetti da LES. Syk è, altresì, coinvolta nell'attivazione delle cellule mesangiali con conseguenti produzione di citochine proinfiammatorie a livello glomerulare e proliferazione cellulare. Dati preliminari hanno dimostrato un suo potenziale ruolo nel controllo dei meccanismi fisiopatologici della glomerulonefrite a depositi di IgA. Uno studio di fase II sta valutando l'efficacia e la sicurezza del fostamatinib nel trattamento di questa nefropatia (NCT02112838).

\section{Blocco della co-stimolazione linfocitaria}

L'Abatacept è una proteina di fusione (composta dal dominio extracellulare di CTLA-4 e dal frammento Fc di IgG1) che, legandosi al CD80 (B7-1) delle cellule presentanti l'antigene, inibisce una completa attivazione dei linfociti T, la loro proliferazione e la produzione di citochine infiammatorie. Alcuni studi sperimentali hanno evidenziato l'efficacia di questo farmaco nel trattamento del LES; in particolare la sua associazione con la ciclofosfamide ha consentito di ottenere una riduzione significativa della proteinuria, del titolo anticorpale e della mortalità in un modello murino di nefrite lupica (39). Uno studio pubblicato recentemente, pur non avendo dimostrato una riduzione del numero di flare in pazienti con LES trattati con Abatacept, ha evidenziato miglioramenti significativi della qualità di vita degli stessi. Un altro recente trial di fase II/III che ha coinvolto pazienti con nefrite lupica non ha raggiunto l'end-point primario (risposta renale completa valutata come: 1 ) valore di filtrato con variazioni non superiori al $10 \%$ rispetto ai valori basali, 2) escrezione urinaria proteine/creatinina ratio $<0.26 \mathrm{mg} / \mathrm{mg}, 3$ ) sedimento inattivo) (40).

Tuttavia, la rivalutazione degli stessi dati utilizzando altri indici di outcome ha evidenziato effetti favorevoli del trattamento con Abatacept.

L'Abatacept ha suscitato notevole interesse anche nel trattamento delle "patologie renali con proteinuria". Yu et al (41) hanno recentemente proposto una nuova classificazione per queste patologie dividendo le forme B7-1 pos/neg. La presenza di B7-1, normalmente assente, sui podociti riduce la loro capacità di legarsi alla matrice attraverso la B1-integrina e cambia le loro caratteristiche morfologiche e le loro funzioni, portando a un distacco dei pedicelli dalla membrana basale glomerulare e causando proteinuria. Partendo da questo presupposto, gli Autori hanno trattato pazienti con sindrome nefrosica severa secondaria a glomerulosclerosi focale e segmentaria primitiva o post-trapianto, positivi per 
B7-1 con Abatacept, ottenendo dei buoni risultati. Sulla base delle stesse premesse, il farmaco è stato impiegato anche nel trattamento della glomerulonefrite a lesioni minime. Questi risultati preliminari non hanno, al momento, trovato sostanziali conferme.

L'Abatacept è stato proposto anche per il trattamento delle vasculiti ANCA-correlate. In particolare, la sua efficacia è stata valutata in 20 pazienti con recidive non severe di GPA (42); in questa coorte, ha consentito una remissione completa in 16/20 pazienti e un mantenimento della stessa per una durata di 14.4 mesi in 14 pazienti. È, attualmente, in corso uno studio di fase III (ABROGATE) per la conferma degli effetti clinici positivi dell'Abatacept nel trattamento delle vasculiti.

Altra modalità di blocco della co-stimolazione linfocitaria è rappresentata dall'impiego dell'anticorpo anti-CD40L. II CD40L è over-espresso nei modelli murini di lupus e la sua inattivazione attraverso l'anticorpo monoclonale è risultata efficace nel trattamento della nefrite lupica degli stessi modelli. Sono stati condotti 2 trial in pazienti con LES trattati con anti-CD40L (IDEC-131 e BG9588). II primo studio non ha evidenziato miglioramenti clinici significativi rispetto al placebo dopo 20 settimane. Nel secondo trial, invece, sono stati rilevati una riduzione del titolo degli anticorpi anti-DNA e un incremento dei livelli del complemento. Lo studio, tuttavia, è stato interrotto precocemente per un numero inatteso di eventi tromboembolici.

\section{Blocco della cascata infiammatoria}

I farmaci biologici rivolti a inibire l'azione delle principali citochine coinvolte nel sistema infiammatorio, come il TNF- $\alpha$, I'IL-1 o I'IL-6, per anni sono stati utilizzati con buoni risultati in ambito reumatologico e gastroenterologico. Più recentemente, sono stati proposti anche in nefrologia. Si pensa che il TNF $\alpha$ abbia un ruolo patogenetico nelle vasculiti ANCA-correlate. Alcuni studi non controllati hanno riportato effetti favorevoli del trattamento delle vasculiti con anti-TNF $\alpha$. Questi risultati, tuttavia, non sono stati confermati dal WGET-trial, in cui la terapia con anti-TNF $\alpha$ non ha consentito il mantenimento della remissione clinica nelle granulomatosi sistemiche. L'efficacia di questo gruppo di farmaci nel trattamento delle vasculiti, al momento, rimane dubbia.

L'Adalimumab, anticorpo monoclonale anti-TNF $\alpha$, è stato proposto per il trattamento delle GSFS (24) In particolare, un trial pubblicato nel 2010 ha evidenziato un miglioramento significativo della proteinuria in $4 / 10$ pazienti trattati con questo farmaco. L'Adalimumab è stato valutato anche nel trattamento delle micropoliangioiti sistemiche (43). Lo studio di fase II, finalizzato a valutare l'efficacia e la sicurezza del farmaco come terapia aggiuntiva nei pazienti con vasculiti, ha fornito dei risultati promettenti sia in termini di remissione che di mantenimento della stessa. Questi dati, tuttavia, dovranno essere confermati da casistiche più ampie.

L'IL-6, una citochina con doppio effetto pro-infiammatorio e antinfiammatorio, è coinvolta nella patogenesi della nefrite lupica. In un modello murino, l'IL-6 esogena è in grado di aumentare la produzione di autoanticorpi e di accelerare la progressione del danno renale. Nei pazienti con LES, i livelli di IL-6 si correlano con l'attività clinica e il titolo degli Ab anti-DNA. Negli stessi pazienti, l'escrezione urinaria di IL-6 è risultata superiore e si riduce dopo il trattamento. Tocilizumab è un anticorpo monoclonale diretto contro il recettore dell'IL- 6 in grado di prevenire il legame sia con la forma solubile del recettore che con quella legata alla membrana cellulare. In uno studio recente (44) di fase 1 , che ha valutato 16 pazienti con un'attività moderata di malattia trattati con Tocilizumab, il farmaco ha consentito una riduzione dei livelli dei marker infiammatori e degli autoanticorpi, mentre i valori della proteinuria sono rimasti invariati. Nello stesso studio, però, la maggior parte dei pazienti ha sviluppato una neutropenia dose-correlata ed è stato registrato un numero elevato di complicanze infettive. Saranno a disposizione a breve i risultati di un trial che valuta l'efficacia e la sicurezza del trattamento con Tocilizumab nel LES.

Un'altra alternativa proposta per il trattamento del LES è il Sirukumab, anticorpo monoclonale che lega I'IL-6 riducendone l'attività biologica. II farmaco è stato precedentemente valutato in uno studio di fase 1 che non ha fornito risultati definitivi (45). È attualmente in corso un trial che valuta la sua efficacia nella terapia della nefrite lupica (NCT01273389).

Molti studi hanno fatto emergere un ruolo importante del tumor necrosis factor (TNF)-like weak (TWEAK, TNFSF12) nella patogenesi della nefrite lupica. II ruolo di TWEAK nel favorire la risposta infiammatoria, la proliferazione delle cellule renali, l'apoptosi, le alterazioni vascolari e la fibrosi, insieme al riscontro di elevate concentrazioni nei pazienti con nefrite lupica, ha portato a ipotizzare un ruolo attivo di questa citochina nei pazienti con LES e coinvolgimento renale. L'impiego di BIIBO, anticorpo monoclonale anti-TWEAK, nella nefrite lupica (classe III o IV) è in corso di valutazione nell'ATLAS-Study (Clinical trial NCT01499355).

Altri studi/trial in corso riguardano l'utilizzo di anticorpi anti-INF $\alpha$ come il Rontalizumab o anti IFN- $\gamma$ (NCT00818948).

Un'ulteriore alternativa di blocco della cascata infiammatoria potrebbe essere rappresentata dalla deplezione sistemica dei macrofagi. La proteina di fusione CCL2-LPM, legandosi al recettore di membrana espresso sui macrofagi, viene internalizzata consentendo l'inattivazione dei ribosomi e la sintesi proteica con conseguente morte cellulare. Questa molecola si è dimostrata efficace in un modello animale di glomerulonefrite proliferativa mesangiale. La sua efficacia nel trattamento delle glomerulonefrite a depositi di IgA è in corso di valutazione (NCT00856674). Ulteriori studi sono necessari per valutare le possibili applicazioni nel trattamento delle nefropatie.

\section{Conclusioni}

Negli ultimi vent'anni, i farmaci biologici hanno cambiato lo scenario terapeutico in molte specialità mediche compresa la 
nefrologia. Le potenzialità di questi agenti sono ancora in fase di sviluppo. Tra i punti incompletamente risolti dell'impiego di queste molecole ci sono la sicurezza nel lungo periodo e il costo/beneficio. È verosimile che, con il tempo, si possa prevedere un sostanziale impulso all'ottimizzazione della produzione e I'utilizzo di queste terapie nella pratica clinica quotidiana.

\section{Disclosures}

Financial support: No financial support was received for this submission.

Conflict of interest: The authors have no conflict of interest.

\section{Bibliografia}

1. Roccatello D. An innovative approach to the treatment of immune-mediated glomerular diseases. G Ital Nefrol 2013; 30(1):1-9.

2. Rood IM, Hofstra JM, Deegens JK, Wetzels JF. B cell suppression in primary glomerular disease. Adv Chronic Kidney Dis 2014; 21(2):166-81.

3. Jones RB. Rituximab in the treatment of anti-neutrophil cytoplasm antibody-associated vasculitis. Nephron Clin Pract 2014;128 (3-4):243-9.

4. Roccatello $D$, Vangelista $A$, Pani $A$. The role of rituximab in the treatment of ANCA-associated systemic vasculitis. G Ital Nefrol 2011;28(5):474-88.

5. Jones RB, Tervaert JW, Hauser T, et al. Rituximab versus cyclophosphamide in ANCA-associated renal vasculitis. N Engl J Med 2010;363(3):211-20.

6. Geetha $\mathrm{D}$, Specks $U$, Stone $J H$, et al. Rituximab versus cyclophosphamide for ANCA-associated vasculitis with renal involvement. Rituximab for ANCA-Associated Vasculitis Immune Tolerance Network Research Group. J Am Soc Nephrol 2015; 26(4):976-85.

7. Tanna A, Pusey C. Clinical trials. Rituximab for maintenance of remission in AAV. Nat Rev Nephrol 2015;11(3):131-2.

8. Beckwith H, Lightstone L. Rituximab in Systemic Lupus Erythematosus and Lupus Nephritis. Nephron Clin Pract 2014; 128: 250-4.

9. Roccatello D, Sciascia S, Rossi D, etal. Intensive short-term treatment with rituximab, cyclophosphamide and methylprednisolone pulses induces remission in severe cases of SLE with nephritis and avoids further immunosuppressive maintenance therapy. Nephrol Dial Transplant 2011;26(12):3987-92.

10. Merrill JT, Neuwelt CM, Wallace DJ, et al. Efficacy and safety of rituximab in moderately-to-severely active systemic lupus erythematosus: the randomized, double-blind, phase II/II systemic lupus erythematosus evaluation of rituximab trial. Arthritis Rheum 2010;62(1):222-33.

11. Rovin BH, Furie R, Latinis K et al. Efficacy and safety of rituximab in patients with active proliferative lupus nephritis: the Lupus Nephritis Assessment with Rituximab study. Arthritis Rheum 2012;64(4):1215-26.

12. Weidenbusch M, Rommele C, Schrottle A, Anders HJ. Beyond the LUNAR trial. Efficacy of rituximab in refractory lupus nephritis. Nephrol Dial Transplant 2013;28:106-11.

13. Moroni G, Raffiotta F, Trezzi B, et al. Rituximab vs mycophenolate and vs cyclophosphamide pulses for induction therapy of active lupus nephritis: a clinical observational study. Rheumatology 2014;53(9):1570-7.

14. Condon MB, Ashby D, Pepper RJ, et al. Prospective observational single-centre cohort study to evaluate the effectiveness of treating lupus nephritis with rituximab and mycophenolate mofetil but no oral steroids. Ann Rheum Dis 2013;72:1280-6.

15. Roccatello D, Sciascia S, Baldovino S, et al. A 4-year observation in lupus nephritis patients treated with an intensified B-lymphocyte depletion without immunosuppressive maintenance treatmentClinical response compared to literature and immunological re-assessment. Autoimmun Rev 2015;14(12):1123-30.

16. De Vita S, Quartuccio L, Isola M, et al. A randomized controlled trial of rituximab for the treatment of severe cryoglobulinemic vasculitis. Arthritis Rheum 2012;64(3):843-53.

17. Roccatello $D$, Baldovino $S$, Rossi $D$, et al. Long-term effects of anti-CD20 monoclonal antibody treatment of cryoglobulinaemic glomerulonephritis. Nephrol Dial Transplant 2004;19(12): 3054-61.

18. Quartuccio L, Zuliani F, Corazza L, et al. Retreatment regimen of rituximab monotherapy given at the relapse of severe HCVrelated cryoglobulinemic vasculitis: Long-term follow up data of a randomized controlled multicentre study. J Autoimmun 2015;63:88-93.

19. Cravedi P, Remuzzi G, Ruggenenti P. Rituximab in primary membranous nephropathy: first-line therapy, why not? Nephron Clin Pract 2014;128(3-4):261-9.

20. Irazabal MV, Eirin A, Lieske J, et al. Low- and high-molecularweight urinary proteins as predictors of response to rituximab in patients with membranous nephropathy: a prospective study. Nephrol Dial Transplant 2013;28(1):137-46.

21. Rojas-Rivera J, Fernández-Juárez G, Ortiz A, et al. A European multicentre and open-label controlled randomized trial to evaluate the efficacy of Sequential treatment with TacrolimusRituximab versus steroids plus cyclophosphamide in patients with primary Membranous Nephropathy: the STARMEN study. Clin Kidney J 2015;8(5):503-10.

22. Kronbichler $A$, Bruchfeld $A$. Rituximab in adult minimal change disease and focal segmental glomerulosclerosis. Nephron Clin Pract 2014;128(3-4):277-82.

23. Janardan J, Ooi K, Menahem S. Sustained complete remission of steroid- and cyclophosphamide-resistant minimal-change disease with a single course of rituximab therapy. Clin Kidney J 2014;7(3):293-5.

24. Malaga-Dieguez L, Bouhassira D, Gipson D, Trachtman H. Novel therapies for FSGS: preclinical and clinical studies. Adv Chronic Kidney Dis 2015;22(2):e1-6.

25. Javaugue V, Karras A, Glowacki F, et al. Long-term kidney disease outcomes in fibrillary glomerulonephritis: a case series of 27 patients. Am J Kidney Dis 2013;62(4):679-90.

26. Syeda UA, Singer NG, Magrey M. Anti-glomerular basement membrane antibody disease treated with rituximab: A casebased review. Semin Arthritis Rheum 2013;42(6):567-72.

27. Mysler EF, Spindler AJ, Guzman R, et al. Efficacy and safety of ocrelizumab in active proliferative lupus nephritis: results from a randomized, double-bind, phase III. Arthritis Rheum 2013; 65(9):2368-79.

28. Al Rayes H, Touma Z. Profile of epratuzumab and its potential in the treatment of systemic lupus erythematosus. Drug Des Devel Ther 2014;8:2303-10.

29. Wallace DJ, Goldenberg DM. Epratuzumab for systemic lupus erythematosus. Lupus 2013;22(4):400-5.

30. Wallace DJ, Goldenberg DM. Epratuzumab for patients with moderate to severe flaring SLE: health-related quality of life outcomes and corticosteroid use in the randomized controlled ALLEVIATE trials and extension study SL0006. Rheumatology 2014;53(3):502-11.

31. Strand V, Petri M, Kalunian K, et al. ClinicalTrials.gov. Identifier NCT01262365, a phase 3, randomized, double-blind, placebocontrolled, multicenter study of the efficacy and safety of four 12 -week treatment cycles (48 weeks total) of epratuzumab in 
systemic lupus erythematosus subjects with moderate to severe disease (EMBODY 1); $14^{\text {th }}$ Dec 2010 - [cited 30th March 2014].

32. Navarra SV, Guzman RM, Gallacher AE, et al. Efficacy and safety of belimumab in patients with active systemic lupus erythematosus: a randomized, placebo-controlled, phase 3 trial. Lancet 2011;377:721-31.

33. Furie $\mathrm{R}$, Petri $\mathrm{M}$, Zamani $\mathrm{O}$, et al. A phase III, randomized, placebo-controlled study of belimumab, a monoclonal antibody that inhibits B lymphocyte stimulator, in patients with systemic lupus erythematosus. Arthritis Rheum 2011;63:3918-30.

34. Lenert $A$, Lenert P. Current and emerging treatment options for ANCA-associated vasculitis: potential role of belimumab and other BAFF/APRIL targeting agents. Drug Des Devel Ther 2015;9:333-47.

35. Isenberg DA, Petri M, Kalunian K, et al. Efficacy and safety of subcutaneous tabalumab in patients with systemic lupus erythematosus: results from ILLUMINATE-1, a 52-week, phase III, multicentre, randomised, double-blind, placebo-controlled study. Ann Rheum Dis 2015.

36. Ginzler EM, Wax S, Rajeswaran A, et al. Atacicept in combination with MMF and corticosteroids in lupus nephritis: results of a prematurely terminated trial. Arthritis Res Ther 2012;14(1):R33.

37. Ghoreschi K, Gadina M. New small molecules in autoimmune and inflammatory diseases. Exp Dermatol 2014;23(1):7-11.

38. Morales-Torres J. The status of fostamatinib in the treatment of rheumatoid arthritis. Expert Rev Clin Immunol 2012;8(7):609-15.
39. Cunane G, Chan OT, Cassafer G, et al. Prevention in renal damage in murine lupus nephritis by CTLA-4Ig and cyclophosphamide. Arthritis Rheum 2004;50:1539-48.

40. Furie $R$, Nicholis $K$, Cheng TT, et al. Efficacy and safety of abatecept over 12 months in patients with lupus nephritis: results from a multicenter, randomized, double blind, placebo-controlled phase II/III study. Arthritis Rheum 2011; 63:S962-3.

41. Yu CC, Fornoni A, Weins A, et al. Abatacept in B7-1-positive proteinuric kidney disease. N Engl J Med 2013;369(25):2416-23.

42. Langford CA, Monach PA, Specks U, et al. An open-label trial of abatacept (CTLA4-IG) in non-severe relapsing granulomatosis with polyangiitis (Wegener's). Vasculitis Clinical Research Consortium. Ann Rheum Dis 2014;73(7):1376-9.

43. Silva-Fernández L, Loza E, Martínez-Taboada VM, et al. Biological therapy for systemic vasculitis: a systematic review. Systemic Autoimmune Diseases Study Group of the Spanish Society for Rheumatology (EAS-SER). Semin Arthritis Rheum 2014;43(4):542-57.

44. Szepietowski JC, Nilganuwong S, Wozniacka A, et al. Phase I Randomized, Double-Blind, Placebo-Controlled, Multiple intravenous, Dose-Ascending Study of Sirukumab in Cutaneous or Systemic Lupus Erythematosus. Arthritis Rheum 2013;65:2661-71.

45. Rajadhyaksha AG, Mehra S, Nadkar MY. Biologics in SLE: current status. J Assoc Physicians India 2013;61(4):262-7. 